\title{
QUEEN'S
UNIVERSITY
BELFAST
}

\section{High resolution Thomson Parabola Spectrometer for full spectral capture of multi-species ion beams}

Alejo, A., Kar, S., Tebartz, A., Ahmed, H., Astbury, S., Carroll, D. C., Ding, J., Doria, D., Higginson, A., McKenna, P., Neumann, N., Scott, G. G., Wagner, F., Roth, M., \& Borghesi, M. (2016). High resolution Thomson Parabola Spectrometer for full spectral capture of multi-species ion beams. Review of Scientific Instruments, 87(8), [083304]. https://doi.org/10.1063/1.4961028

Published in:

Review of Scientific Instruments

Document Version:

Publisher's PDF, also known as Version of record

Queen's University Belfast - Research Portal:

Link to publication record in Queen's University Belfast Research Portal

\footnotetext{
Publisher rights

Copyright 2016 AIP Publishing.

This article may be downloaded for personal use only. Any other use requires prior permission of the author and AIP Publishing.

The following article appeared in Alejo, A, Kar, S, Tebartz, A, Ahmed, H, Astbury, S, Carroll, DC, Ding, J, Doria, D, Higginson, A, McKenna,

P, Neumann, N, Scott, GG, Wagner, F, Roth, M \& Borghesi, M 2016, 'High resolution Thomson Parabola Spectrometer for full spectral capture of multi-species ion beams' Review of Scientific Instruments, vol 87, no. 8, 083304 and may be found at

(http://scitation.aip.org/content/aip/journal/rsi/87/8/10.1063/1.4961028

\section{General rights}

Copyright for the publications made accessible via the Queen's University Belfast Research Portal is retained by the author(s) and / or other copyright owners and it is a condition of accessing these publications that users recognise and abide by the legal requirements associated
} with these rights.

Take down policy

The Research Portal is Queen's institutional repository that provides access to Queen's research output. Every effort has been made to ensure that content in the Research Portal does not infringe any person's rights, or applicable UK laws. If you discover content in the Research Portal that you believe breaches copyright or violates any law, please contact openaccess@qub.ac.uk. 


\section{AIP $\mid \begin{aligned} & \text { Review of } \\ & \text { Scientific Instruments }\end{aligned}$}

\section{High resolution Thomson Parabola Spectrometer for full spectral capture of multi- species ion beams}
A. Alejo, S. Kar, A. Tebartz, H. Ahmed, S. Astbury,
D. C. Carroll, J. Ding,
D. Doria, A. Higginson, P. McKenna , N. Neumann, G. G. Scott, F. Wagner, M. Roth, and M. Borghesi

Citation: Review of Scientific Instruments 87, 083304 (2016); doi: 10.1063/1.4961028

View online: http://dx.doi.org/10.1063/1.4961028

View Table of Contents: http://scitation.aip.org/content/aip/journal/rsi/87/8?ver=pdfcov

Published by the AIP Publishing

\section{Articles you may be interested in}

High-energy resolution Thomson Parabola spectrometer for laser plasma diagnostics

AIP Conf. Proc. 1546, 50 (2013); 10.1063/1.4816605

Thomson spectrometer-microchannel plate assembly calibration for MeV-range positive and negative ions, and neutral atoms

Rev. Sci. Instrum. 84, 053302 (2013); 10.1063/1.4803670

Calibration of a Thomson parabola ion spectrometer and Fujifilm imaging plate detectors for protons, deuterons, and alpha particles

Rev. Sci. Instrum. 82, 073301 (2011); 10.1063/1.3606446

High energy ion beam analysis of buried $\alpha-\mathrm{Fe} 2 \mathrm{O} 3$ (0001) /a- Al 2 O 3 (0001) interface AIP Conf. Proc. 576, 432 (2001); 10.1063/1.1395341

Modified Thomson spectrometer for the detection of low energy ( $\mathrm{keV}$ ) high power ion beams Rev. Sci. Instrum. 68, 3738 (1997); 10.1063/1.1148018

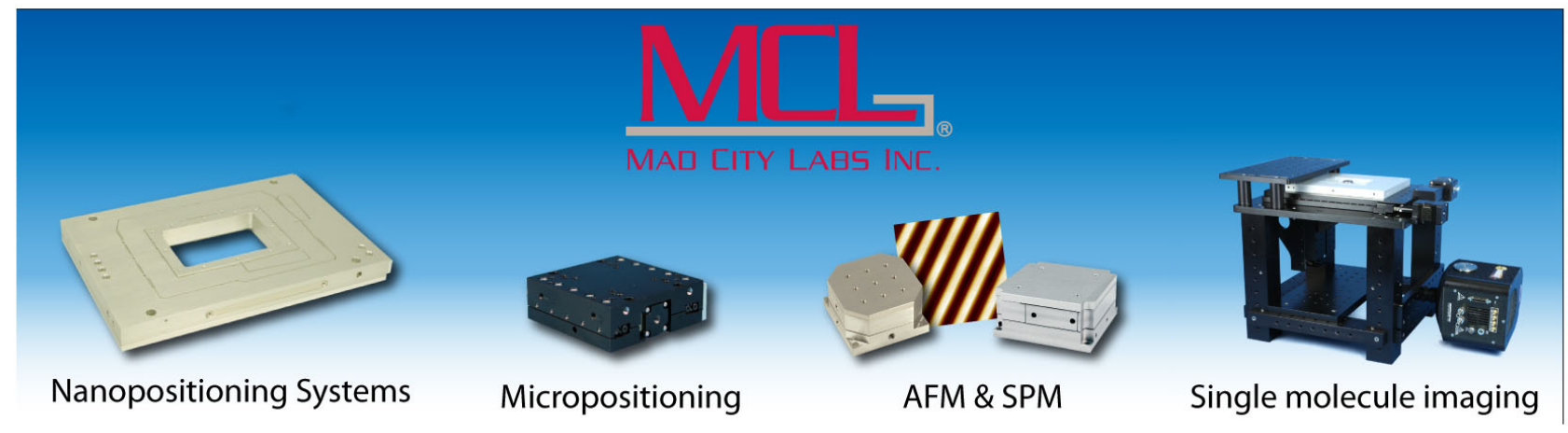




\title{
High resolution Thomson Parabola Spectrometer for full spectral capture of multi-species ion beams
}

\author{
A. Alejo, ${ }^{1}$ S. Kar, ${ }^{1, a)}$ A. Tebartz, ${ }^{2}$ H. Ahmed, ${ }^{1}$ S. Astbury,${ }^{3}$ D. C. Carroll, ${ }^{3}$ J. Ding, ${ }^{2}$ D. Doria,${ }^{1}$ \\ A. Higginson, ${ }^{4}$ P. McKenna, ${ }^{4}$ N. Neumann, ${ }^{2}$ G. G. Scott, ${ }^{3}$ F. Wagner, ${ }^{5}$ M. Roth, ${ }^{6}$ \\ and M. Borghesi ${ }^{1}$ \\ ${ }^{1}$ Centre for Plasma Physics, School of Mathematics and Physics, Queen's University Belfast, \\ Belfast BT7 1NN, United Kingdom \\ ${ }^{2}$ Institut für Kernphysik, Technische Universität Darmstadt, Schloßgartenstrasse 9, \\ D-64289 Darmstadt, Germany \\ ${ }^{3}$ Central Laser Facility, Rutherford Appleton Laboratory, Didcot, Oxfordshire OX11 OQX, United Kingdom \\ ${ }^{4}$ Department of Physics, SUPA, University of Strathclyde, Glasgow G4 ONG, United Kingdom \\ ${ }^{5}$ GSI Helmholtzzentrum für Schwerionenforschung GmbH, Planckstraße 1, 64291 Darmstadt, Germany \\ ${ }^{6}$ Institut für Kernphysik, Technische Universität Darmstadt, Schloßgartenstraße 9, \\ D-64289 Darmstadt, Germany
}

(Received 13 May 2016; accepted 2 August 2016; published online 17 August 2016)

\begin{abstract}
We report on the experimental characterisation of laser-driven ion beams using a Thomson Parabola Spectrometer (TPS) equipped with trapezoidally shaped electric plates, proposed by Gwynne et al. [Rev. Sci. Instrum. 85, 033304 (2014)]. While a pair of extended (30 cm long) electric plates was able to produce a significant increase in the separation between neighbouring ion species at high energies, deploying a trapezoidal design circumvented the spectral clipping at the low energy end of the ion spectra. The shape of the electric plate was chosen carefully considering, for the given spectrometer configuration, the range of detectable ion energies and species. Analytical tracing of the ion parabolas matches closely with the experimental data, which suggests a minimal effect of fringe fields on the escaping ions close to the wedged edge of the electrode. The analytical formulae were derived considering the relativistic correction required for the high energy ions to be characterised using such spectrometer. Published by AIP Publishing. [http://dx.doi.org/10.1063/1.4961028]
\end{abstract}

\section{INTRODUCTION}

Ion acceleration driven by high-power lasers has attracted a considerable interest over the last decade due to a range of potential applications in healthcare, industry, and science. ${ }^{1,2}$ A number of ion acceleration mechanisms, such as Target Normal Sheath Acceleration (TNSA), ${ }^{3-5}$ Radiation Pressure Acceleration (RPA), ${ }^{6-8}$ Break-Out Afterburner (BOA),${ }^{9,10}$ and guided post-acceleration by laser-driven microcoils, ${ }^{11}$ are currently being the subject of intense investigation, which is leading to significant progress towards the $100 \mathrm{MeV} / \mathrm{n}$ range. ${ }^{5}$ These mechanisms inherently produce multi-species ion beams, due to the chemical composition of the target bulk and/or the contamination layer covering the target (typically containing hydrocarbons and water vapour). ${ }^{12}$ Among the ion diagnostics which are commonly used, such as radiochromic film stack, ${ }^{13}$ nuclear activation, ${ }^{14}$ or nuclear track detection, ${ }^{15,16}$ the Thomson Parabola Spectrometer (TPS) ${ }^{17}$ is arguably the most popular diagnostic due to its unique capability of spectrally characterising the ion beams while discriminating species with different charge-to-mass ratio $(Z / A)$.

As with any diagnostic, the resolution of a TPS is a key parameter in view of its use in a laser-driven ion acceleration experiment. In the case of TPSs, two types of resolutions are of interest, viz., the energy resolution along a given trace (ion species), and the $Z / A$ resolution - which defines the ability to

a)Electronic mail: s.kar@qub.ac.uk discriminate two neighbouring ion traces. The $Z / A$ resolution for a given TPS configuration varies across the range of energies resolved by the magnetic deflection, and becomes critical for high-energy ions (tens of $\mathrm{MeV} /$ nucleon). ${ }^{18-20}$

The separation between neighbouring ion traces can be increased by several ways, such as increasing the electric field strength or the length of the electric plates, or moving the detector farther away from the plates. However, as discussed in Gwynne et al. ${ }^{19}$ increasing the length of the electric plates is the most effective route for increasing the $Z / A$ resolution at high energies, while maintaining a compact design that can be fielded within the typical dimensions of an interaction chamber. Therefore, it was proposed in Ref. 19 to implement a pair of extended, trapezoidally shaped electric plates, which will not only resolve ion traces at high energies, but will also retain the lower energy part of the spectrum by avoiding the spectral clipping typically associated to rectangular plates, which becomes progressively more significant as the length of the plates is extended. Although Gwynne et al. ${ }^{19}$ demonstrated experimentally the effectiveness of the extended electric plates, the effect of the trapezoidal electric plates was only explored by particle tracing simulations.

In this paper, we show the experimental characterisation of laser-driven multi-species ion beams employing a TPS with a pair of extended (30 cm-long) trapezoidal plates. The ion traces in the data closely match with the analytical prediction, which suggests negligible fringe field effect despite the unconventional plate shape. Such extended-electrode ion 
spectrometers will be particularly useful for detecting high energy (hundreds of $\mathrm{MeV} /$ nucleon) multispecies ion beams, predicted from experiments employing multi-petawatt lasers in upcoming facilities. ${ }^{21,22}$ For this reason, we implemented a fully relativistic treatment of the ion propagation within the TPS. The remainder of the paper is organised as follows. Sec. II describes the experimental setup and the design of the trapezoidally shaped electric plates considering the TPS dimensions and species of interest. The experimental results are discussed in Sec. III, and the relativistic analytical formula describing the shape of the ion traces on the detector is discussed in Sec. IV.

\section{EXPERIMENTAL SETUP AND DESIGN OF THE TRAPEZOIDAL PLATES}

The experiment was carried out at the Rutherford Appleton Laboratory (RAL), STFC, UK by employing the petawatt arm of the VULCAN laser system. ${ }^{23}$ The data presented in this paper were obtained by irradiating the laser onto $25 \mu \mathrm{m}$ thick aluminium foil targets. Using an $f / 3$ off-axis parabolic mirror, the laser was focussed down to $\sim 6 \mu \mathrm{m}$ full width at half-maximum spots on the target, delivering a peak intensity in excess of $10^{20} \mathrm{~W} \mathrm{~cm}^{-2}$. The TPS was placed at a distance of $\sim 106 \mathrm{~cm}$ along the target normal direction, where the maximum ion energies are expected from TNSA, the main acceleration mechanism for the conditions of the experiment. ${ }^{24}$

A schematic side view of a TPS with trapezoidal electric plates is shown in Fig. 1. A pinhole at the entrance of the diagnostic selects an ion pencil beam, which travels through regions of parallel magnetic $\left(B_{0}\right)$ and electric $\left(E_{0}\right)$ fields, both transverse to the beam axis. The magnetic field determines the position of the ions along the $y$-axis at the detector plane, depending on their energy per nucleon, while the electric field deflects the ions along the $x$-axis according to their chargeto-mass $(Z / A)$ ratios. Considering homogeneous electric and magnetic fields, the position of the ions reaching the detector can be analytically derived (see the Appendix) as

$$
x=\frac{\gamma}{\gamma^{2}-1} \frac{q E_{0} L_{E}}{m_{0} c^{2}}\left(T_{E}-L_{E} / 2\right),
$$

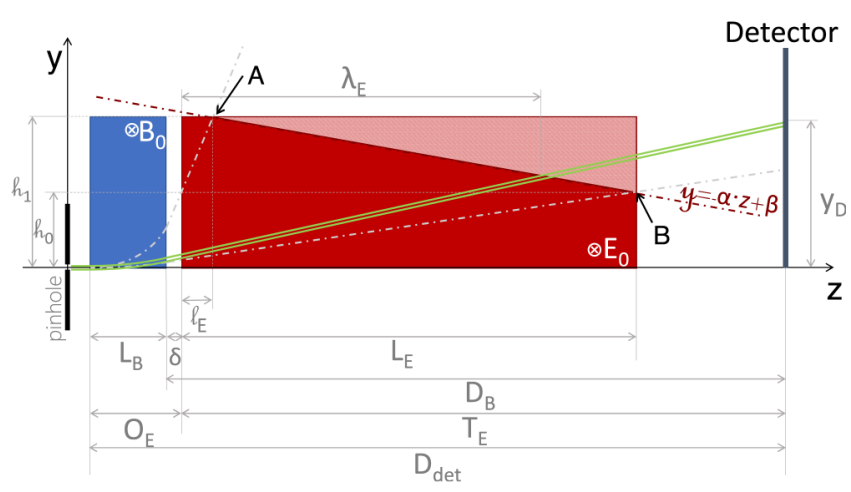

FIG. 1. Schematic side view of a Thomson parabola spectrometer using trapezoidal electric plates.

$$
y=\frac{q B_{0} L_{B}}{m_{0} c \sqrt{\gamma^{2}-1}}\left(L_{B} / 2+D_{B}\right),
$$

where $q$ and $m_{0}$ are the charge and rest mass of the species of interest, $\gamma$ is the Lorentz factor, and $L_{B}, D_{B}, T_{E}$, and $L_{E}$ are the dimensions of relevant sections of the TPS, as labelled in Fig. 1.

The line $y=-\alpha z+\beta$, where $\alpha=\left(h_{1}-h_{0}\right) /\left(L_{E}-l_{E}\right)$ and $\beta=\left[h_{1}\left(L_{E}-O_{E}\right)-h_{0}\left(l_{E}+O_{E}\right)\right] /\left(L_{E}-l_{E}\right)$, defines the line along which the rectangular electrodes were cut to obtain the trapezoidal shape. In order to find the optimum trapezoidal design for the electric plates, the locations at which different energy ions would impinge on the negatively biased $\left(-V_{b}\right)$ electrode were calculated for each of the relevant ion species. For a given separation between the beam axis and the negative electrode $(\Delta)$, the clipping position $(x, y, z)=\left(\Delta, y_{e}^{\text {clip }}, L_{e}^{\text {clip }}\right)$ can be expressed as

$$
\begin{aligned}
& L_{e}^{c l i p}=\sqrt{\frac{\gamma^{2}-1}{\gamma} \frac{2 \Delta m_{0} c^{2}}{q E_{0}}}, \\
& y_{e}^{c l i p}=\frac{q B_{0} L_{B}}{m_{0} c \sqrt{\gamma^{2}-1}}\left(O_{E}-L_{B} / 2+L_{e}^{c l i p}\right) .
\end{aligned}
$$

In the experiment discussed here, we were interested in the characterisation of protons and carbon ions $\left(\mathrm{C}^{\{4-6\}+}\right)$, whose impinging position is plotted in Fig. 2(a). These curves were obtained for the TPS setup used in our experiment, i.e., using $B_{0} \simeq 0.972 \mathrm{~T}, E_{0} \simeq 20 \mathrm{kV} / \mathrm{cm}, L_{B}=50 \mathrm{~mm}, O_{E}=75 \mathrm{~mm}$, $L_{E}=300 \mathrm{~mm}, \Delta \simeq 7 \mathrm{~mm}$, and $D_{d e t}=493 \mathrm{~mm}$. For the usual rectangular plates (shown by the black dashed line in Fig. 2(a)), a significant range of ion energies would be blocked by the $-V_{b}$

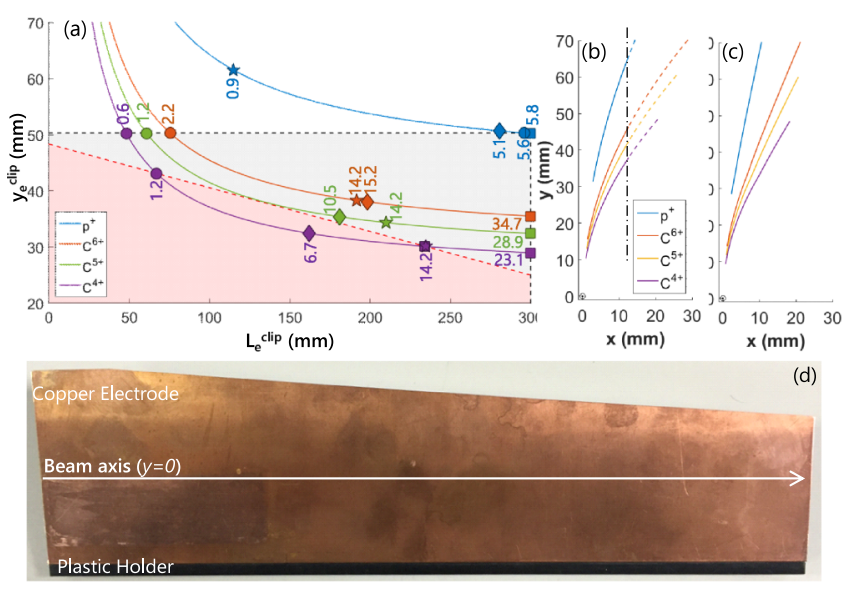

FIG. 2. (a) Graph plotting the positions on the plane of the $-V_{b}$ electrode (depth, $L_{e}^{\text {clip }}$, and height, $y_{e}^{\text {clip }}$ ) where ions would impinge depending on their $\mathrm{Z}, \mathrm{A}$, and energy. The shape of the $30 \mathrm{~cm}$-long rectangular electrode used in the experiment is shown by the black dashed line. The red dashed line represents the line along which the rectangular plate was cut to produce the trapezoidally shaped (red shaded area) electrode. The different markings on the curves represent the minimum (filled $\circ$ ) and maximum (filled $\square$ ) energies of ions clipped by the plates, the detection threshold due to the stopping in the IP filter (filled $\star$ ), and the finite detector size (filled $\diamond$ ). The energy values in $\mathrm{MeV}$ are shown next to each marking. (b) and (c) show analytically plotted ion traces on the detector while using the $30 \mathrm{~cm}$-long rectangular electrodes and the derived trapezoidal plates, respectively. Dashed lines in (b) correspond to the region of the traces being clipped by the rectangular plates. (d) Picture of one of the trapezoidal electrodes used in the TPS during the experiment. 

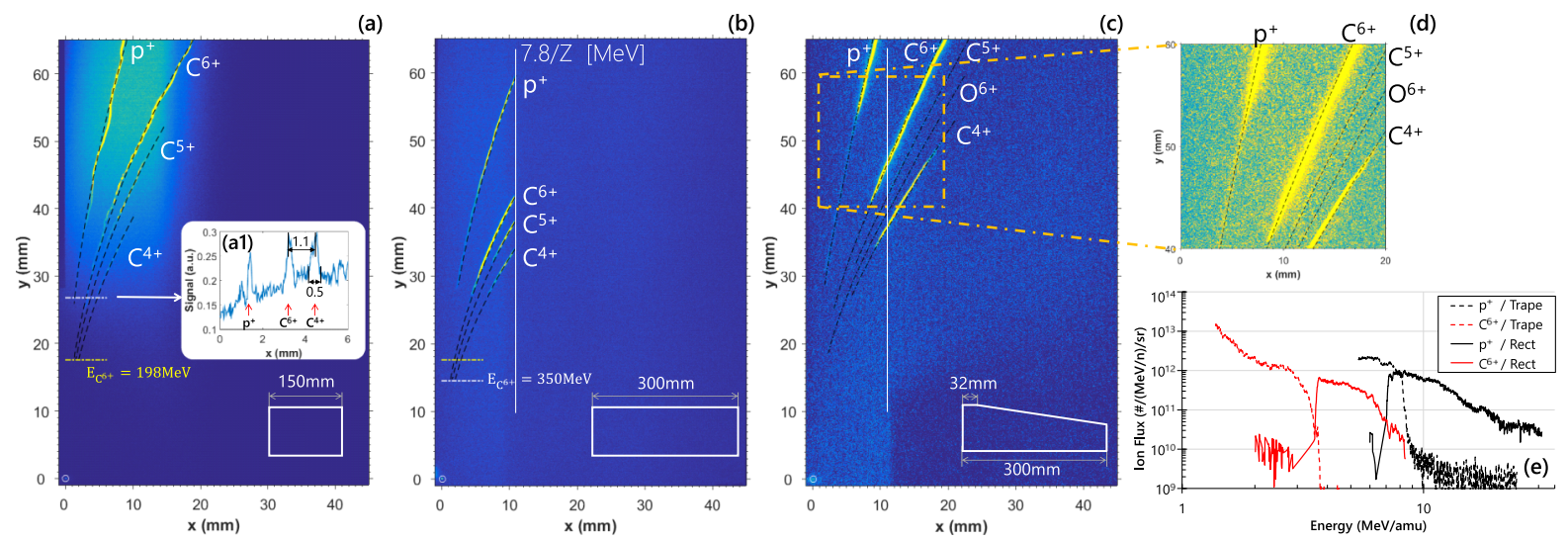

FIG. 3. ((a)-(c)) Experimental raw data from the IP scans using different designs of the electric plates, as drawn in the bottom right corner of each image. Black dashed lines show the fit to the ion traces using the analytical formulae mentioned in the text. (a1) shows a line-out across the ion traces along the white dashed line in (a). The white vertical line in (b) shows the position of the sharp cut-off in the ion traces shadowed by the electrode. (d) Zoomed-in view of a part of the data shown in (c), as marked by the orange dashed square. (e) Spectra of proton (black) and carbon (red) ions retrieved from the TPS using the rectangular (solid line) and trapezoidal (dashed) electric plates.

electrode, which would produce partially clipped traces on the detector as shown in Fig. 2(b). Therefore, the modified design involves cutting off a section of the electrode, shown by the gray area in Fig. 2(a), so that the curves for all the species of interest lie above the electrode.

However, one has to be careful not to remove a larger section of the plate than necessary. The range of ion energies that one aims to unblock by creating a trapezoidal electrode shape depends on the given TPS setup. The minimum ion energy for a given species detectable by the detector depends on two factors, such as the finite size of the detector and the use of any filtering in front of the detector. Since Image Plate (IP) detectors were used in our case, which are sensitive to ambient lighting, the common practice is to wrap the IPs with a thin $\mathrm{Al}$ foil to avoid exposure to ambient light before the IP is scanned after the shot. The clipping position on the electrode for the lower energy cut-off for different ion species, due to either our detector size $(70 \mathrm{~mm} \times 45 \mathrm{~mm})$ or the stopping in the IP filter, is marked respectively as " $\diamond$ " and " $\star$ " in Fig. 2(a). For each species, the minimum detectable energy would be the rightmost of the two points-whereas the detector size is the limiting factor for $\mathrm{p}^{+}$and $\mathrm{C}^{6+}$, the filtering defines the cut-off energy for the $\mathrm{C}^{4+}$ and $\mathrm{C}^{5+}$ species.

With the aforementioned considerations, the final plate design was chosen, as shown by the red shaded area in Fig. 2(a), which would allow the entire detectable range of energies and species to reach the detector, producing unclipped traces as plotted in Fig. 2(c). The trapezoidal shaped electrodes thus used in the experiment were manufactured from a $1 \mathrm{~mm}$-thick copper sheet, mounted on a plastic holder as shown in Fig. 2(c).

In the experiment, the TPS was fielded with BAS-TR imaging plates as detectors, which were wrapped with $12 \mu \mathrm{m}$ thick Al foil to avoid accidental exposure to ambient light after the irradiation. The IPs were scanned 60-90 min after the irradiation, using a commercial IP scanner (Fujifilm FLA$5000^{25}$ ), with a 16-bit dynamic range and a $25 \mu \mathrm{m} \times 25 \mu \mathrm{m}$ pixel size. The measured signal was converted from the scan value (or quantum level (QL)) to PSL using the formula given by the manufacturer, ${ }^{20,26}$ and then was corrected for the fading of the signal due to spontaneous decay. ${ }^{20,27}$

\section{EXPERIMENTAL RESULTS}

In the experiment, three electrode designs were tested to compare the results obtained with each of them on a proof-ofprinciple basis. Initially, a TPS with $150 \mathrm{~mm}$-long rectangular electric plates was used to characterise the laser-driven ion beams, producing the data shown in Fig. 3(a).

Although the $\mathrm{C}^{6+}$ and $\mathrm{C}^{4+}$ traces in this shot were well separated, this set-up would have been ineffective in discriminating these traces at the higher energies expected from optimized targets, advanced mechanisms and/or at higher power laser facilities. The maximum $\mathrm{C}^{6+}$ energy that can be distinguished from the neighbouring traces in the current TPS would have been $198 \mathrm{MeV}(16.5 \mathrm{MeV} /$ nucleon). It is to be noted that, due to the limitations in laser performance encountered during this campaign, the maximum proton and carbon energies obtained from this shot were $28 \mathrm{MeV}$ and $102 \mathrm{MeV}$ respectively, which are significantly less than what can be obtained from the TNSA mechanism, ${ }^{4}$ or from ultra-thin (sub-micron) targets at such laser systems. ${ }^{7}$

The electrodes were exchanged for $300 \mathrm{~mm}$-long rectangular electric plates in the following step (Fig. 3(b)), keeping the same $O_{E}$ distance. It can be clearly seen by comparing the data shown in Figs. 3(a) and 3(b), how the separation between the different traces at the high-energies was enlarged. The improvement in the trace separation, and the possibility of discriminating traces at high energies (around $100 \mathrm{MeV} /$ nucleon), can be seen from the simulated traces, which matches well with the experimental data for lower energies. The only issue with such setup is, however, the clipping of the lower energy part of the ion traces by the extended electrodes, which appears as a sharp vertical cut-off of the data as shown in Fig. 3(b). A precise measurement of the distance between the ion-beam axis and the $-V_{b}$ electrode $(\Delta)$ can be obtained from the clipping position, $\Delta=6.7 \pm 0.5 \mathrm{~mm}$, which agrees with the measurement taken during the setup.

Finally, the electrodes were replaced by the trapezoidal plates, made according to the optimised design discussed in Sec. II. The experimental result obtained using these trapezoidal electrodes is shown in Fig. 3(c). As expected, the 
separation between the traces in this case matches exactly with that obtained with the rectangular long plates, but without the low energy clipping. Where the finite size of the detector limits the minimum detectable energy for species such as $\mathrm{p}^{+}$or $\mathrm{C}^{6+}$, the $\mathrm{C}^{4+}$ trace faded within the detector field of view due to the stopping of the low energy ions inside the IP filter. One can also find the presence of faint traces of $\mathrm{C}^{5+}$ and $\mathrm{O}^{6+}$, as shown in the zoomed-in view in Fig. 3(d), which were well separated. Unfortunately the ion energy in this shot was too low, again due to shot-to-shot variation in laser performance during the campaign, to show a set of well-separated, unclipped traces extending to higher energies. However, the data clearly show the expected advantage arising from the use of trapezoidal electrodes.

\section{COMPARISON BETWEEN EXPERIMENTAL AND ANALYTICAL TRACES}

While employing trapezoidal electric plates, the part of the trace formed by the ions passing over the electrode does not follow the same parabolic curve defined by the higher energy part of the trace. This is due to the lower energy ions effectively experiencing the electric field region over a different propagation length than the higher energy ions. Therefore, such design would not be useful without an analytical method to retrieve the ion spectra. Assuming $\lambda_{E}$ to be the length of the electric field region experienced by a given ion, the position of the ion on the detector can be written as (by substituting $\lambda_{E}$ in place of $L_{E}$ in Eq. (1))

$$
x=\frac{\gamma}{\gamma^{2}-1} \frac{q E_{0} \lambda_{E}}{m_{0} c^{2}}\left(T_{E}-\lambda_{E} / 2\right) .
$$

The variable $\lambda_{E}$ is given by the $z$-position at which the particle exits the electric-field region, and therefore can be expressed as

$$
\lambda_{E}=\left\{\begin{array}{ll}
\frac{\beta+L_{B} \cdot \Phi}{\alpha+2 \Phi}-O_{E}, & E_{\text {lim }}^{A} \leq E \leq E_{\text {lim }}^{B}, \\
L_{E}, & E \geq E_{\text {lim }}^{B}
\end{array},\right.
$$

where $\alpha$ and $\beta$ define the line along which the electrodes were cut (Fig. 1), and the dimensionless factor $\Phi$ is given by $\Phi$ $=q B_{0} L_{B} /\left(2 m_{0} c \sqrt{\gamma^{2}-1}\right)$. The limits $E_{\text {lim }}^{A}$ and $E_{\text {lim }}^{B}$ define the energy range for which the ions exit the electric field region through the chopped side of the plate, corresponding to the points A and B in Fig. 1, respectively. For energies higher than $E_{\text {lim }}^{B}$, the particles will leave the plates through the longest region, experiencing a constant plate length of $L_{E}$ independent of their energy. On the other end, for energies below $E_{\text {lim }}^{A}$, the Larmor radius will cause the particles to leave the electric field through the top flat section of the plates. The values for both energy limits can be derived as shown in Eq. (7),

$$
\begin{aligned}
\frac{E_{l i m}^{A}}{m_{0} c^{2}} & =\sqrt{\left[\frac{q B_{0} L_{B}\left[O_{E}+l_{E}-L_{B} / 2\right]}{m_{0} c h_{1}}\right]^{2}+1}-1, \\
\frac{E_{l i m}^{B}}{m_{0} c^{2}} & =\sqrt{\left[\frac{q B_{0} L_{B}\left[O_{E}+L_{E}-L_{B} / 2\right]}{m_{0} c h_{0}}\right]^{2}+1}-1 .
\end{aligned}
$$

The ion traces on the detector for the TPS with trapezoidally shaped electrodes can then be defined by using the formulae in Eqs. (5) and (2). These formulae are based on the approximation of a homogeneous electric field inside the region between the two trapezoidal electrodes and zero electric field elsewhere, i.e., ignoring the fringe fields appearing close to the wedged-side of the electrodes. However, the ion traces derived using Eqs. (5) and (2), depicted by the dashed lines in Figs. 3(c) and 3(d), show a good agreement with the experimental traces. Therefore, it is reasonable to ignore any fringe effects around the plates for the range of energies of concern.

Although the ion traces produced by the TPS with trapezoidal electrodes do not follow a parabolic trajectory in the lower energy range, one can identify the ion species corresponding to a given trace by either considering the parabolic part of the trace or by using the modified TPS formulae given by Eqs. (5) and (2). Once the ion trace is identified and isolated from the rest of the data, one can obtain the ion spectrum on the basis of the magnetic field deflection and the detector calibration. As an example, the $\mathrm{p}^{+}$and $\mathrm{C}^{6+}$ spectra obtained from the data shown in Figs. 3(b) and 3(c) are shown in Fig. 3(e). As it can be seen here, using a trapezoidal shaped electrode allowed detecting the lower-energy part of the ion spectrum, which would be otherwise clipped when using a rectangular shaped electrode. The capability of detecting lower energy ions may also be valuable as a monitoring tool for failed or low efficiency shots, where obtaining some spectral information may aid understanding the causes for the low performance in accelerating ions.

\section{CONCLUSIONS}

The ongoing advances in laser-driven ion acceleration, coupled with the prospect of significant progress at upcoming laser facilities, are leading to the need for compact ion spectrometers for characterisation of multi-species, high energy ion sources, capable of resolving ion species at high energies (10s-100 MeV/nucleon). Here we have presented the experimental demonstration of a modified TPS design with trapezoidally shaped electrodes, proposed by Gwynne et al. ${ }^{19}$ The method for designing an optimised electrode shape for our experimental setup is discussed, along with an analytical formulation for simulating the ion traces produced by such TPS. The analytically plotted ion traces match closely with the experimental data, proving that fringe-field effects on the ions escaping over the wedged side of the trapezoidal electrodes are not significant. The proposed analytical treatment takes consideration of the relativistic corrections required for very high energy ( $\gtrsim 100 \mathrm{MeV} /$ nucleon) ions, expected in the near future from interactions involving multi-petawatt lasers at upcoming facilities.

\section{ACKNOWLEDGMENTS}

The authors acknowledge funding from EPSRC [Nos. EP/J002550/1-Career Acceleration Fellowship held by S.K., EP/L002221/1, EP/K022415/1, and EP/J500094/1]. The authors also acknowledge the support of the mechanical engineering staff of the Central Laser Facility, STFC, UK. Data 
associated with the research published in this paper can be accessible at http://dx.doi.org/10.17034/d721c24c-7c68-4be49716-418e62c85165.

\section{APPENDIX: ANALYTICAL DERIVATION OF THE TPS EQUATIONS}

The trajectory of ions passing through a TPS is solved using Newton's laws. In order to obtain the parabolic shape of the ion traces observed on the detector, ${ }^{19,20}$ a low magnetic field approximation is commonly used, in which the Larmor radius $\left(R_{L}\right)$ is large enough $\left(\gg L_{B}\right)$ to consider $v_{y} \ll v_{z}$.

In case of a relativistic particle of rest mass $m_{0}$, charge $q$, and kinetic energy $U$ entering a TPS, the acceleration experienced by the particle can be written as

$$
\vec{a}=\frac{1}{m_{0} \gamma(\vec{v})}[\vec{F}-(\vec{\beta} \cdot \vec{F}) \vec{\beta}],
$$

where $\gamma$ is the Lorentz factor and $\vec{F}=q(\vec{E}+\vec{v} \times \vec{B})$ is the Lorentz force experienced by the particle inside the homogeneous electric and magnetic fields.

Since the energy gain from the electric field is negligible $\left(\Delta U \leq Z e V_{0} \ll m_{0} c^{2}\right), \vec{\beta}$ and $\gamma=1+U / m_{0} c^{2}$ can be assumed to be constant during its transit inside the TPS diagnostic. The term $\vec{\beta} \cdot \vec{F}$ in Eq. (A1) for different regions inside the TPS can be expressed as

$$
\vec{\beta} \cdot \vec{F} \sim\left\{\begin{array}{ll}
\vec{\beta} \cdot \vec{E}=\beta_{x} E_{0} \ll E_{0} & \vec{E}=E_{0} \hat{x}, \vec{B}=0 \\
\vec{\beta} \cdot(\vec{\beta} \times \vec{B})=0 & \vec{E}=0, \vec{B}=B_{0} \hat{x} \\
0 & \vec{B}=\vec{E}=0
\end{array} .\right.
$$

Therefore, for a relativistic particle diagnosed by a TPS, the acceleration can simply be expressed as Newton's law with a correction for the inertial mass,

$$
\vec{a}=\frac{1}{m_{0} \gamma} \vec{F} .
$$

The low magnetic field approximation mentioned above also upholds for the relativistic particles, as the condition, $R_{L}=m_{0} c \sqrt{\gamma^{2}-1} / q B_{0} \gg L_{b}$ remains valid for the typical parameters (few Tesla magnetic field strength extended over tens of centimetres) used in experiments.

Under these considerations, the equations of motion along different axes can be written analytically as

$$
\begin{aligned}
& x=\frac{q E_{0} L_{E}}{\gamma m_{0} v_{z}^{2}}\left(T_{E}-L_{E} / 2\right), \\
& y=\frac{q B_{0} L_{B}}{\gamma m_{0} v_{z}}\left(L_{B} / 2+D_{B}\right),
\end{aligned}
$$

Using $\gamma v_{z}=c \sqrt{\gamma^{2}-1}$ and $\gamma v_{z}^{2}=c^{2} \cdot\left(\gamma^{2}-1\right) / \gamma$, one can rewrite the equations as shown in Eqs. (1) and (2).

\footnotetext{
${ }^{1}$ A. Macchi, M. Borghesi, and M. Passoni, Rev. Mod. Phys. 85, 751 (2013). ${ }^{2}$ H. Daido, M. Nishiuchi, and A. S. Pirozkhov, Rep. Prog. Phys. 75, 056401 (2012).
}

${ }^{3}$ J. Fuchs, P. Antici, E. d'Humières, E. Lefebvre, M. Borghesi, E. Brambrink, C. Cecchetti, M. Kaluza, V. Malka, M. Manclossi et al., Nat. Phys. 2, 48 (2005).

${ }^{4}$ L. Robson, P. Simpson, R. Clarke, K. Ledingham, F. Lindau, O. Lundh, T. McCanny, P. Mora, D. Neely, C.-G. Wahlström et al., Nat. Phys. 3, 58 (2006).

${ }^{5}$ F. Wagner, O. Deppert, C. Brabetz, P. Fiala, A. Kleinschmidt, P. Poth, V. Schanz, A. Tebartz, B. Zielbauer, M. Roth, T. Sthlker, and V. Bagnoud, Phys. Rev. Lett. 116, 205002 (2016).

${ }^{6}$ A. Robinson, M. Zepf, S. Kar, R. Evans, and C. Bellei, New J. Phys. 10, 013021 (2008).

${ }^{7}$ S. Kar, K. Kakolee, B. Qiao, A. Macchi, M. Cerchez, D. Doria, M. Geissler, P. McKenna, D. Neely, J. Osterholz et al., Phys. Rev. Lett. 109, 185006 (2012).

${ }^{8}$ J. Bin, W. Ma, H. Wang, M. Streeter, C. Kreuzer, D. Kiefer, M. Yeung, S. Cousens, P. Foster, B. Dromey et al., Phys. Rev. Lett. 115, 064801 (2015).

${ }^{9}$ L. Yin, B. Albright, B. Hegelich, and J. Fernández, Laser Part. Beams 24, 291 (2006).

${ }^{10}$ L. Yin, B. Albright, K. Bowers, D. Jung, J. Fernández, and B. Hegelich, Phys. Rev. Lett. 107, 045003 (2011).

${ }^{11}$ S. Kar, H. Ahmed, R. Prasad, M. Cerchez, S. Brauckmann, B. Aurand, G. Cantono, P. Hadjisolomou, C. L. Lewis, A. Macchi et al., Nat. Commun. 7, 10792 (2016).

${ }^{12}$ S. Gitomer, R. Jones, F. Begay, A. Ehler, J. Kephart, and R. Kristal, Phys. Fluids 29, 2679 (1986).

${ }^{13}$ F. Nürnberg, M. Schollmeier, E. Brambrink, A. Blažević, D. Carroll, K. Flippo, D. Gautier, M. Geissel, K. Harres, B. Hegelich et al., Rev. Sci. Instrum. 80, 033301 (2009).

${ }^{14}$ R. Clarke, P. Simpson, S. Kar, J. Green, C. Bellei, D. Carroll, B. Dromey, S. Kneip, K. Markey, P. McKenna et al., Nucl. Instrum. Methods Phys. Res., Sect. A 585, 117 (2008).

${ }^{15}$ R. Fleischer, P. Price, and R. Walker, J. Appl. Phys. 36, 3645 (1965).

${ }^{16}$ S. Kar, M. Borghesi, L. Romagnani, S. Takahashi, A. Zayats, V. Malka, S. Fritzler, and A. Schiavi, J. Appl. Phys. 101, 044510 (2007).

${ }^{17}$ J. J. Thomson, Proc. R. Soc. London, Ser. A 89, 1 (1913).

${ }^{18}$ D. Carroll, P. Brummitt, D. Neely, F. Lindau, O. Lundh, C.-G. Wahlström, and P. McKenna, Nucl. Instrum. Methods Phys. Res., Sect. A 620, 23 (2010).

${ }^{19}$ D. Gwynne, S. Kar, D. Doria, H. Ahmed, M. Cerchez, J. Fernandez, R. Gray, J. Green, F. Hanton, D. MacLellan, P. McKenna, Z. Najmudin, D. Neely, J. Ruiz, A. Schiavi, M. Streeter, M. Swantusch, O. Willi, M. Zepf, and M. Borghesi, Rev. Sci. Instrum. 85, 033304 (2014).

${ }^{20}$ A. Alejo, S. Kar, H. Ahmed, A. Krygier, D. Doria, R. Clarke, J. Fernandez, R. Freeman, J. Fuchs, A. Green, J. Green, D. Jung, A. Kleinschmidt, C. Lewis, J. Morrison, Z. Najmudin, H. Nakamura, G. Nersisyan, P. Norreys, M. Notley, M. Oliver, M. Roth, J. Ruiz, L. Vassura, M. Zepf, and M. Borghesi, Rev. Sci. Instrum. 85, 093303 (2014).

${ }^{21}$ D. Papadopoulos, C. Le Blanc, G. Chériaux, P. Georges, G. Mennerat, J. P. Zou, F. Mathieu, and P. Audebert, Advanced Solid State Lasers (Optical Society of America, 2013), paper ATu3A.43.

${ }^{22}$ Extreme light infraestracture(eli), http://www.eli-np.ro//.

${ }^{23}$ C. Danson, P. Brummitt, R. Clarke, J. Collier, B. Fell, A. Frackiewicz, S. Hancock, S. Hawkes, C. Hernandez-Gomez, P. Holligan, M. Hutchinson, A. Kidd, W. Lester, I. Musgrave, D. Neely, D. Neville, P. Norreys, D. Pepler, C. Reason, W. Shaikh, T. Winstone, R. Wyatt, and B. Wyborn, Nucl. Fusion 44, S239 (2004).

${ }^{24}$ S. Wilks, A. Langdon, T. Cowan, M. Roth, M. Singh, S. Hatchett, M. Key, D. Pennington, A. MacKinnon, and R. Snavely, Phys. Plasmas 8, 542 (2001).

${ }^{25}$ Fujifilm bas-tr imaging plates, http://www.fujifilm.com.

${ }^{26}$ Information about conversion of ip scanner readout to psl, http://beamline. harima.riken.jp/b145xu/web_old/Info/BAS2500imgSpec.pdf.

${ }^{27}$ D. Doria, S. Kar, H. Ahmed, A. Alejo, J. Fernandez, M. Cerchez, R. J. Gray, F. Hanton, D. A. MacLellan, P. McKenna, Z. Najmudin, D. Neely, L. Romagnani, J. A. Ruiz, G. Sarri, C. Scullion, M. Streeter, M. Swantusch, O. Willi, M. Zepf, and M. Borghesi, Rev. Sci. Instrum. 86, 123302 (2015). 